\title{
CREENCIAS EPISTEMOLÓGICAS Y POSIBILIDADES DE CAMBIO EN ESTUDIANTES DE FÍSICA DE 10 GRADO
}

\section{Resumen}

El presente artículo muestra un avance de la investigación: "Creencias y Posibilidades de Cambio Epistemológico en estudiantes de Física de Décimo Grado" que los integrantes del grupo INVAUCOL vienen desarrollando en dos colegios oficiales del Distrito Capital con apoyo del CIUP-UPN y Colciencias. Presenta algunos de los referentes teóricos sobre los cuales se basa el trabajo y plantea unos resultados preliminares relacionados con las creencias que mantienen los estudiantes que participan en la investigación en relación con la noción de movimiento. Por último, presenta en forma general las estrategias de cambio epistemológico que se están implementando.

Palabras clave: Creencias, aprendizaje, física, epistemología, movimiento.

\begin{abstract}
The present work shows an advance of the investigation: "Beliefs and Possibilities of Change Epistemologic in students of Physics of Tenth Grade" that the members of the group INVAUCOL comes developing in two official high schools of the Capital District with support of the CIUP-UPN and Colciencias. It presents references theoretical some about the work is based and it outlines some preliminary results related with the beliefs that the students and participate in the investigation in connection with the movement notion maintain. Lastly, it presents in general form the strategies of change epistemologic that are implementing.
\end{abstract}

Key words: Beliets, students, learning, physics, epistemologic, movement.

\footnotetext{
1 Docente investigador, Universidad Pedagógica Nacional. Director Grupo Investigación por las aulas colombianas. Invaucol - invaucol@hotmail.com 2 Docente investigador, Colegio INEM de Kennedy.

${ }^{3}$ Docente Investigador, Colegio Heladia Mejía. Catedrático UPN.

${ }^{4}$ Estudiante de física de la UPN.

5 Docente investigador, Colegio INEM de Kennedy.

${ }^{6}$ Docente investigador, Colegio INEM de Kennedy.
} 


\section{Presentación}

En el marco de las tendencias alternativas en la investigación sobre el pensamiento y el conocimiento de los estudiantes, este proyecto indaga por las posibilidades de cambio epistemológico y su incidencia en la enseñanza y el aprendizaje de conceptos específicos. Concretamente, el proyecto se desarrolla con estudiantes de décimo grado de dos colegios oficiales de Bogotá.

En los estudios de casos se pregunta por la naturaleza, el tipo y la función de los referentes epistemológicos que mantienen algunos estudiantes de física; igualmente se interroga por la posibilidad de favorecer un cambio en las creencias epistemológicas de dichos estudiantes. Así mismo, se estudia si dicho cambio favorece el aprendizaje y la enseñanza del concepto de movimiento.

Como proceso general se han desarrollado tres instrumentos de obtención de ideas previas acerca de la noción de movimiento; a saber:

A. El primer instrumento consiste en una escala Liker. Para la construcción de esta escala se tuvieron en cuenta cuatro paradigmas en física, históricamente significativos: paradigma aristotélico, paradigma parisino o del ímpetus, paradigma clásico y paradigma contemporáneo.

De cada uno de estos paradigmas se seleccionaron tres principios fundantes que marcan la diferencia entre uno y otro. De esta manera, la escala quedó conformada por 15 proposiciones. El rango de valor de la escala es: 1. Completamente de acuerdo; 2 . De acuerdo; 3 . Inseguro; 4. En desacuerdo; 5 . Completamente en desacuerdo.

La escala fue piloteada en un estudio previo que se llevó a cabo en tres grupos de estudiantes de 10 grado, de 30 estudiantes cada uno.
B. El segundo instrumento consiste en un cuestionario abierto. La construcción de este instrumento presupone la explicitación de 6 problemas relevantes para identificar posibles rupturas históricas en la comprensión de la noción de movimiento.

Los criterios de diferenciación de los paradigmas epistemológicos que subyacen a la noción de movimiento que mantienen los estudiantes, toman dos aspectos: los tipos de razonamiento (de sentido común y matemático) y el objeto de la física (mundo físico o principios matemáticos), según los cuatro paradigmas de la física que el grupo pretende identificar en las ideas previas de los estudiantes.

C. El tercer instrumento consiste en un protocolo abierto de entrevista. Este instrumento es una estrategia para explicitar los tipos de razonamiento que frecuentemente presentan los estudiantes y la manera como ellos conciben el problema del movimiento.

De la aplicación de los instrumentos se obtuvo información acerca de las ideas previas que algunos estudiantes de 10 grado mantiene sobre el movimiento.

En este artículo presentamos unos primeros resultados sobre las ideas previas de los estudiantes a partir de la aplicación de la escala Liker. Igualmente se pone en consideración las estrategias que el grupo está implementando para favorecer el cambio epistemológico. En posteriores avances presentaremos los resultados de esta implementación.

\section{Marco de referencia}

Es de amplio reconocimiento, en el campo de la didáctica de las ciencias naturales y sociales, que el estudiante llega al salón de clases con ideas previas (Driver, 1986; Viennot, 1985) acerca de las nociones que se pretenden enseñar por parte del profesor. En los últimos 25 años, es amplio el número de artículos publicados en los que se conceptualiza, identifica y caracteriza multiplicidad de ideas previas, al mismo tiempo que se analiza e interpreta la incidencia de estas ideas en el aprendizaje de los estudiantes. Sólo en el campo de la física, en un valioso trabajo de revisión bibliográfica que ha realizado Elgin Wolfe ${ }^{7}$, él ha identificado 126 artículos dedicados desde 1988 a este tema, igualmente ha identificado 98 en el ámbito de las ideas previas en biología y 89 en el ámbito de la química. Lo anterior sin contar con la gran cantidad de artículos publicados en revistas de habla hispana como Enseñanza de las Ciencias e Investigación en la Escuela.

Igualmente, es conocida la tesis según la cual "el aprendizaje no se produce de forma automática a partir de la enseñanza. Tiene lugar principalmente a través del procesamiento activo y esforzado de la información por parte de los alumnos, los cuales deben percibir e interpretar las acciones de los docentes para que éstas influyan en su rendimiento." (Wittrock, 1990:543). Dicho procesamiento tiene lugar también en el ámbito de las creencias epistemologías que mantienen los estudiantes; es decir, que el procesa-

\footnotetext{
7 Wolfe es profesor de la Universidad de Toronto, ha realizado un valioso trabajo de identificación y clasificación de artículos publicados en; Physics Teacher; Physics Education; Journal of Research in Science Teaching: Journal of Science education; Contemporary Educational Psychology; Science Education; Research in Science and Technological Education; Instructional Science; American Journal of Physics; School Science and Mathematic; Journal of Chemical Education; Journal of Biological Education; American Biology Teacher; entre otras revistas de habla inglesa. Este amigo ha puesto a nuestra disposición este rico material que aún se encuentra inédito. Wolfe se refiere a las ideas previas de la siguiente manera: "The word "misconception" refers to a person's understanding of a concept that differs in significant ways from the commonly accepted scientific understanding of the concept. "Alternate conception" is a kinder term that means roughly the same thing. Another possible term is "naive conception". An example misconception is described below".
} 
miento de la información por parte del estudiante no se da sólo a nivel de las estructuras de pensamiento o nivel cognoscitivo que lo constituyen, sino también a nivel de los contenidos específicos de dicho pensamiento. Así, las creencias epistemologías del estudiante constituyen un marco de referencia desde la cual ellos interpretan y comprenden las teorías expuestas por el profesor.

Ahora bien, las ideas previas que mantienen los estudiantes sobre una misma noción pueden diferir en su naturaleza, dado un perfil epistemológico diverso implícito en dicha noción. Es decir, el estudiante puede llegar al aula atribuyendo múltiples sentidos a una misma noción. Por ejemplo, el estudiante puede, llegar al salón con diferentes creencias acerca de los que es el movimiento o la fuerza. Esos sentidos diferentes pueden estar relacionados con los distintos paradigmas epistemológicos en los cuales la noción de movimiento ha sido pensada. Existiría en las ideas previas que lleva el estudiante al aula, un perfil epistemológico disperso.

\section{Historia y epistemología de la noción de movimiento en la física}

La historia del pensamiento científico presenta grosso modo, cuatro etapas referidas, a su vez, a cuatro tipos de pensamiento. Primero física aristotélica; a continuación física del ímpetus, en tercer lugar la física matemática, experimental, arquimediana o galileana (Cf. Koyré, 1996: 6) y la última la física contemporánea, cuántica y relativista (Cf. Bachelard, 1975). Esos cuatro momentos corresponden, por lo tanto, a cuatro epistemologías distintas, pues como lo ha mencionado el propio Koyré, apoyándose en las tesis bachelardianas, esas etapas corresponden posiblemente a cuatro mutaciones espirituales: "de lo que se trataba no era de combatir unas teorías erróneas, o insuficientes, sino de transformar el marco de

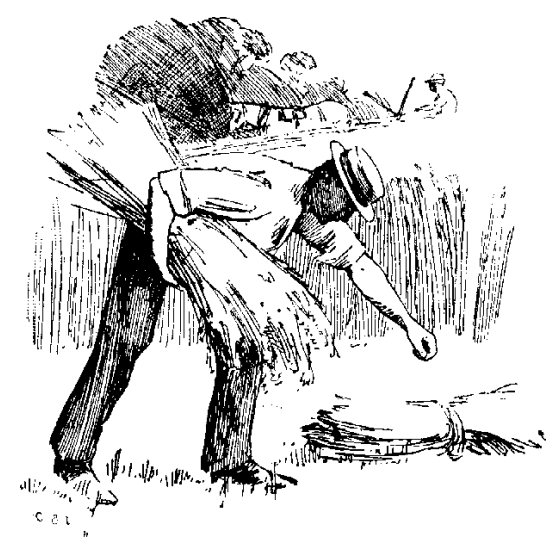

rior significa que la teoría física de Aristóteles aparece como un todo sistemático que debe ser entendido en su unidad intrínseca, antes de pasar a realizar una aproximación que muestre sus debilidades. El sistema aristotélico, por ingenuo que pueda aparecer al físico contemporáneo, se encuentra altamente organizado y es coherente en cierta lógica, lo que hace que sea una representación viable para el sujeto de sentido común. Tal viabilidad es una razón para que las nociones aristotélicas del mundo sean resistentes al cambio cuando pasan a ser las ideas previas de un sujeto. Si bien el pensamiento aristotélico aparece ante los físicos contemporáneos como una explicación ingenua del mundo, también lo es que dicho pensamiento puede ayudarnos a comprender cómo las ingenuidades de "sentido común" pueden constituir un todo coherente y sistemático al interior de sus límites, lo que las hace resistentes y persistentes. Dicha persistencia se da no sólo a nivel de las representaciones mantenidas por los sujetos en la vida cotidiana, como comprensión de su mundo, sino también, y de manera importante, en el ámbito de las explicaciones y comprensiones que mantienen las comunidades científicas en particular y académicas en general, entre estas últimas las comunidades de maestros y estudiantes.

Una evidencia relacionada con la coherencia interna del sistema teórico aristotélico puede encontrarse en la obra de Koyré, según la cual: "la distinción (aristotélica) de los movimientos en 'naturales' y 'violentos' se enmarca en una concepción general de la realidad física" (Koyré, 1996: 8-9). Así, la clasificación y definición del movimiento en naturales y violentos, relativamente ingenua a la luz de los conocimientos contemporáneos, aparece en Aristóteles como una consecuencia necesaria de su concepción de la realidad física. Es la noción de realidad física la que determina las definiciones posteriores de movimiento en Aristóteles.. 
La noción de realidad física en Aristóteles, podría resumirse como una creencia en la existencia de un cosmos, es decir la creencia en la existencia de principios de orden en virtud de los cuales el conjunto de los entes reales forma un todo (naturalmente) bien ordenado. Así, la noción de realidad del sentido común aristotélico aparece como una idea de orden natural. Los principios de esa concepción son:

1. La "intuición fundamental del sentido común" como actitud natural.

2. La creencia en la existencia de "naturalezas" bien determinadas.

3. La creencia en la existencia de un cosmos.

En ese orden de ideas la distinción de los movimientos en "naturales" y "violentos" que hace Aristóteles, se enmarca en una concepción general de la realidad física, la cual se apoya en los principios 2 y 3 mencionados en el párrafo anterior. Así, la creencia en la existencia de un cosmos u orden natural lleva a concebir la existencia necesaria de un lugar natural para cada cosa: "Cada cosa posee en el universo un lugar propio conforme a su naturaleza" (Koyré, 1996: 9). De esa manera los cuerpos pesados que vemos caer lo hacen porque su lugar natural (al cual tienden por naturaleza) es la tierra o centro del mundo y los cuer-

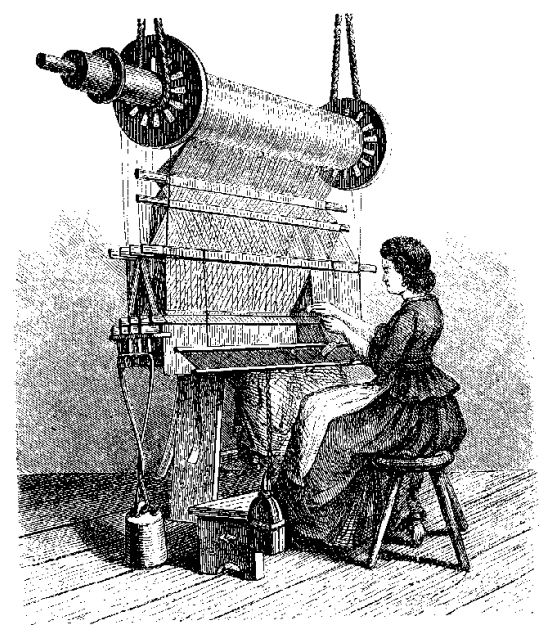

pos livianos se elevan porque su lugar natural es la esfera del último cielo; a todos los movimientos que conducen a los cuerpos a su lugar natural los denomina Aristóteles movimiento natural. En consecuencia, Aristóteles plantea que cuando los cuerpos son sacados de su lugar natural ocurre lo que él denomina un movimiento violento. Según Koyré "la noción de 'lugar natural' traduce una concepción del orden puramente estática" (Koyré, 1996: 9).

En ese sentido, según Koyré de la concepción aristotélica de mundo se deriva necesariamente la concepción según la cual "todo movimiento implica un desorden cósmico, una ruptura del equilibrio, ya sea efecto directo de tal ruptura, causada por la aplicación de una fuerza exterior (violencia), o, por el contrario, efecto del esfuerzo compensador del ser para recuperar su equilibrio perdido y violado, para llevar las cosas a su lugar natural, conveniente, donde podrían reposar y descansar. Este retorno al orden es, justamente, lo que constituye aquello que hemos llamado movimiento natural" (1996: 9). Siendo así las cosas, de la noción de "lugar natural" se desprende también la idea de que el movimiento sea necesariamente un estado pasajero; el movimiento natural se detiene naturalmente cuando ha alcanzado su meta. Por otra parte, también se desprende de la noción de "lugar natural" la idea según la cual el movimiento violento, que es concebido como un estado anormal, no puede perdurar, pues todo tiende naturalmente a su estado natural.

Por otra parte, Aristóteles afirma en el libro IV de la Física que "el movimiento más común y principal, aquel que llamamos 'desplazamiento' es un movimiento con respecto al lugar" (Aristóteles, 1995). De lo anterior se deduce nuevamente que lo que entiende el autor por lugar es determinante de lo que, igualmente, él entiende por movimiento. El movimiento de un móvil lo es con respecto a un lugar que le es natural y en el cual su estado natural es estar en reposo. El espacio con respecto al cual existe el movimiento es, entonces, absoluto. Siendo así, aparece como necesario concebir la idea de una causa o motor que haga que el objeto que se encuentra en su lugar natural lo abandone. En efecto, en el sistema lógico de Aristóteles sólo es posible explicar el hecho de que los objetos salgan de su estado natural de reposo si hay un motor, necesariamente externo, que los impulse a ello. Por principio, no es lógico que un movimiento natural pueda alejar al objeto de su estado de reposo, por lo tanto debe existir un motor externo que produzca ese efecto, que es el que produce el movimiento violento. Tal motor debe existir entonces, y además su naturaleza debe necesariamente implicar la potencia de mover, "pues si el movimiento de todas las cosas que son movidas es o natural o contrario a naturaleza y violento, y si todas las cosas cuyo movimiento es violento y contrario a naturaleza están movidas por algo y por otra cosa que ellas mismas, y sí a su vez todas las cosas cuyo movimiento es natural están movidas por algo, tanto las que se mueven por sí mismas como las que se no se mueven por sí, [...], entonces todas las cosas que están en movimiento tienen que ser movidas por algo" (Aristóteles, 1995: 444445).

Ahora bien, el motor y el móvil, según Aristóteles, deben estar en contacto, pues "un moviente no puede mover otra cosa, sea desde sí mismo hacia otra cosa o desde otra hacia sí mismo, a menos que esté en contacto con ella; así pues, es evidente que en el movimiento local no hay nada intermedio entre lo que mueve y lo que es movido" (Aristóteles, 1995: 398). Con estos presupuestos, Aristóteles puede definir en el libro III de la Física el movimiento como la actualidad simultánea de dos potencias: la de mover y la de ser movido.

En general, la epistemología subyacente al proceso de razonamiento de Aristóteles corresponde a una 
reflexión sobre la observación inmediata como principio fundante del conocimiento y a la lógica formal como principio organizador de tales observaciones. Por otra parte, dicha epistemología presupone, como ya ha sido anunciado, la "intuición fundamental del sentido común" como actitud natural para mostrar la validez o no de los razonamientos lógicos. El objeto de la física en este caso son las intuiciones y representaciones fundadas en la observación común de vida cotidiana.

\subsection{Caracterización de la física del ímpetus}

Esta visión se fundamenta en la idea de que el movimiento de un cuerpo es causado por una especie de impulso externo que se impregna al mismo y le imprime una virtud motriz. Este ímpetus es responsable de que un cuerpo pueda continuar su movimiento una vez este ha sigo generado, en particular el cuerpo se mueve en tal situación debido a la combinación entre el motor y propio movimiento del cuerpo.

El concepto de ímpetus no es del todo claro. Para Koyré (1996: 42) el ímpetus se asocia con una consecuencia del análisis del sentido común entre lo que llamamos esfuerzo muscular e impulso. Aún así, un aspecto valioso en esta caracterización de la física del ímpetus es que esta idea, que ya había sido tratada con anterioridad, es mirada por Benedetti desde una nueva óptica. En efecto, la física del ímpetus debe atender a la pregunta sobre el papel del medio en el movimiento de los cuerpos y, ante esto, responde de forma tajante al desvincular la causa motriz del medio sobre el cuerpo en movimiento ${ }^{8}$. Despojada de esta manera la noción de ímpetus de la noción de "acción de medio físico"; es decir, despojada de sus ataduras "ontológicas" se la puede pen-

${ }^{8}$ Koyré plantea que "en la física aristotélica el medio desempeña un doble papel; es a la vez resistencia y motor: la física del ímpetus niega la acción motriz del medio" (Koyré, 1996: 44). sar en un orden de realidad distinta, puede adquirir una valoración epistemológica diferente. En tal sentido se demarcaría incluso una postura epistemológica susceptible de ser diferenciada en un doble perfil: de sentido común y matemática. El movimiento (en un medio uniforme y en reposo; es decir, en un medio imaginario) esta siendo pensado, en este caso, como un concepto matemático, como una realidad matemática y no necesariamente como una realidad física. Para el caso de la resistencia, por ejemplo esta se entiende como variable matemática del movimiento y es producida, engendrada, por el objeto ${ }^{9}$.

Otro de los cambios epistemológicos trascendentales en la física del ímpetus es la manera como se entiende el movimiento de un objeto en razón de su forma, tamaño, posición y peso. En este sentido, Benedetti afirma que "si no se modifica ni la forma (ni en cantidad ni en calidad) ni la posición de esta forma, el movimiento será proporcional a la virtud motriz, que es el peso o la levedad" Koyré (1996:46). Esta proposición tiene una connotación del todo matemática y es aquí donde radicaría la ruptura epistemológica en contraposición con la visión Aristotélica pues esta se basa en el establecimiento de una proporción ${ }^{10}$, directa o inversa, de las virtudes motrices del cuerpo ${ }^{11}$. La ma-

\footnotetext{
9 "y si el cuerpo que es comparado con otro es de la misma gravedad, pero de resistencia menor, será más rápido que este otro en la misma proporción en que su superficie engendre una resistencia menor que la de este" (Koyré, 1996: 46).

10 Benedetti afirma que "el movimiento natural de un cuerpo grave en diferentes medios es proporcional al peso de este cuerpo en los mismos medios" (en Koyré, 1996: 47).

11 En el orden de ideas expuesto anteriormente, cobra pleno sentido la afirmación preliminar de Benedetti, según la cual él va a "comenzar por enunciar algunas cosas muy ciertas que el intelecto conoce de por si"; es decir, que se le presentan al pensamiento sin mediación de la intuición de sentido común o de la "observación inmediata" del mundo físico". A nuestro juicio, es un llamado a comprender que para hablar de su física, correcta o no, hay que obligarse a pensar en "objetos" matemáticos, en relaciones matemáticas y no, necesariamente, en objetos "naturales".
}

tematización entendida desde el establecimiento de relaciones proporcionales entre las virtudes del cuerpo en movimiento es, por tanto, otra de las características de esta física del ímpetus.

En el orden de ideas expuesto anteriormente, cobra pleno sentido la afirmación preliminar de Benedetti, según la cual él va a "comenzar por enunciar algunas cosas muy ciertas que el intelecto conoce de por si”; es decir, que se le presentan al pensamiento sin mediación de la intuición de sentido común o de la "observación inmediata" del mundo físico". A nuestro juicio, es un Ilamado a comprender que para hablar de su física, correcta o no, hay que obligarse a pensar en "objetos" matemáticos, en relaciones matemáticas y no, necesariamente, en objetos "naturales".

De alguna manera, tanto el espacio como el movimiento son pensados, en esta física, con características relativas a la organización racional de la experiencia que se invoca. Sin embargo, no plenamente, pues, a nuestro juicio, algunas cualidades matemáticas son atribuidas a la "naturaleza" del objeto matemático. Por ejemplo, es posible pensar o postular una resistencia como cualidad propia de un "objeto" matemático y a la vez plantear la noción de movimiento como relativa a esa cualidad. Como si el objeto matemático tuviera unas propiedades intrínsecas, independientes, más halla de la organización racional que de él hace el sujeto racional.

\subsection{Caracterización de la ciencia clásica}

En esta visión se hace prioritario entender el movimiento desde una perspectiva imaginaria, abstracta, matemática. Por ello se concibe al intelecto como medio de análisis del movimiento, donde lo real no depende de los sentidos sino de las representaciones que hacemos en nuestra mente sobre el movimiento de los cuerpos. En tal sentido "el 
proceso del cual salió la física clásica consiste en un esfuerzo para racionalizar, o dicho de otra forma, para geometrizar el espacio y matematizar las leyes de la naturaleza" (Koyré, 1996: 87).

Bajo esta mirada para el caso de Galileo, por ejemplo, se desarrolla una nueva física que tiene en cuenta a la matemática y a la abstracción. Por ello, el movimiento aquí se entiende desde las leyes que son deducidas "abstractamente" y donde su origen no se encuentra en la experiencia cotidiana con objetos reales. Es en esta física que la idea de experimento mental tiene un soporte matemático y por ende físico. De acuerdo con Koyré, "Galileo se esfuerza por desarrollar, de manera coherente y completa la dinámica de la 'fuerza impresa' -del ímpetus-, de la que acabamos de hablar ampliamente, y, al mismo tiempo, por llevar hasta el fin la matematización o, mejor aún, la arquimedización de la física" (Koyré, 1996: 5).

La ruptura epistemológica en el concepto del movimiento con esta nueva física consiste, entonces, en la comprensión del movimiento desde una perspectiva geométrica, matemática, en la cual el objeto es geométrico, matemático y no es el del mundo de lo concreto. Para Crombie (1996) pensadores como Gassendi trabajaron desde esta perspectiva y concibieron el movimiento de los cuerpos desde su interpretación geométrica ${ }^{12}$. En tal sentido esta nueva física, entre otras cosas, cuestiona el papel del ímpetus como causa del movimiento de los cuerpos y responde así mismo en forma contundente al negar tal relación,

\footnotetext{
12 Pierre Gassemdi, “publicó así, por primera vez, la afirmación de que el movimiento que un cuerpo tendría a conservar indefinidamente era rectilínea y que un cambio en la velocidad o dirección requería la operación de una causa externa. También él fue el primero en eliminar conscientemente la noción de ímptus como causa del movimiento. Así la completa geometrización de la física se hizo evidente". Crombie (A.C. Crombie: Historia de la ciencia de san agustín a galileo, Siglos XIII-XVII, 1996, p. 145.
}

como lo expresa Crombie (1996: 145) al analizar los resultados de las publicaciones de Gassendi: También él fue el primero en eliminar conscientemente la noción de ímpetus como causa del movimiento. Así la completa geometrización de la física se hizo evidente".

Por tanto, en la física clásica el movimiento se comprende mejor a partir del espacio geométrico que a partir del espacio concreto. "La actitud intelectual de la ciencia clásica podría estar caracterizada por esos dos momentos, por lo demás íntimamente ligados: la geometrización del espacio y la disolución del Cosmos, es decir, la desaparición, en el interior del razonamiento científico, de toda consideración a partir del cosmos; la sustitución del espacio concreto de la física pregalileana por el espacio abstracto de la geometría euclidiana" (Koyré, 1996: 5). Y sólo a los cuerpos abstractos que se ubican en el espacio geométrico se le aplican las leyes del movimiento.

\subsection{Caracterización de la física contemporánea}

Hacia finales del siglo XIX y principios del $X X$, la física se encontraba en uno de sus mejores momentos. La mecánica clásica de Newton constituía el fuerte filosófico y matemático sobre el cual se sostenían todas las explicaciones sobre los fenómenos naturales y, el concepto fundamental de esta visión era el de corpúsculos o partículas que eran el elemento primario del universo. Los corpúsculos newtonianos eran, básicamente, los pensados por los atomistas griegos, sólo que Newton agregó las causas por las cuales se comportaban como lo hacían. Así, se podían distinguir hasta siete tipos de partículas diferentes, cada una de las cuales representaba un campo específico de operación de natura: las positivas y negativas eléctricas, las del calórico que originaban la expansión de los cuerpos con un aumento de temperatura, las de la materia ponderable o pesos atómicos, las de la luz que se reflejaban y rebotaban, aunque también existía la propuesta ondulatoria de la propagación de estas partículas y, las del magnetismo austral y boreal. Todas ellas gobernadas por las fuerzas de atracción y repulsión cuya "acción a distancia" se producía en el medio del éter.

Podían identificarse cuatro grandes ramas de estudio: la teoría cinética de los gases, la teoría unificada de Maxwell de la electricidad y el magnetismo, las leyes universales del movimiento de Newton y, las leyes de la termodinámica. Pero, estas proposiciones no eran suficientes para dar cuenta de las relaciones intra y subatómicas de las partículas. Existían, además, algunos hechos experimentales que cuestionaban fuertemente el mecanicismo como por ejemplo: la emisión de radiación continua de cuerpos negros (un objeto que absorbe toda la radiación que incide sobre él), el efecto fotoeléctrico y la emisión de líneas espectrales de los átomos.

Vino, entonces, la crisis teórica de la física moderna más grande que sacudió desde los cimientos del universo estático newtoniano, hasta sus partículas inertes que se relacionaban con la mágica acción a distancia.

1900 es un año que marca el inicio de esa revolución en el pensamiento científico. Max Planck introdujo el concepto de "cuanto de acción" para comprender la distribución de la radiación de un cuerpo negro. Einstein, en 1905, utilizó este argumento para explicar el efecto fotoeléctrico, mostrando que la energía de una onda luminosa se puede presentar en forma de paquetes de energía, es decir, como fotones que se encuentran cuantizados. Adicionalmente, el mismo Einstein formuló su teoría especial de la relatividad que hacía todo un replanteamiento sobre la concepción newtoniana de espacio y tiempo absolutos.

De aquí en adelante, el panorama de la física cambió por completo. La 
mecánica cuántica comenzó a develar el misterio del comportamiento atómico de la materia y, la teoría de la relatividad relativizó el espacio y el tiempo, convirtiéndolos en un solo ente: el espacio-tiempo relativo que depende del marco de referencia en el cual se encuentre el observador que lo estudia.

En primer lugar, la mecánica cuántica logró entrelazar la teoría corpuscular de la luz con la teoría ondulatoria; mientras que la primera explica los fenómenos de reflexión y refracción, la segunda describe los de interferencia, difracción y de propagación en un medio. Estamos hablando del comportamiento dual de la luz. Por el otro lado, la relatividad einsteniana puso de manifiesto que los acontecimientos en la naturaleza no pueden medirse todos con el mismo tiempo y, que el espacio no está desligado del tiempo, como lo había planteado Newton.

A partir de ese momento, la noción de movimiento tuvo una profunda transformación. Tanto en el ámbito de los gigantescos fenómenos cósmicos como el movimiento de los planetas, la expansión de las galaxias, la explosión de supernovas, como al nivel de los micro acontecimientos por ejemplo, el movimiento de los electrones alrededor del núcleo atómico, las líneas espectrales de los elementos, el movimiento azaroso de las partículas de un gas. Todos ellos tienen como parámetro principal la luz. En este singular concepto se reúnen tres variables esenciales en la física contemporánea para medir el cambio: tiempo, espacio y energía. Ellos constituyen la base sobre la cual se caracteriza el movimiento en el universo. Finalmente, la expresión: velocidad de la luz nos remite a un evento que puede estar sucediendo en los confines del universo o en nuestras diminutas neuronas.

En ese orden de ideas, Bachelard plantea que "la ciencia contemporánea busca multiplicar la extensión de la noción (...) las mecánicas ondulatoria y quántica desarrollan una inducción de muy largo alcance, una inducción que extiende y dialectiza los principios de la ciencia clásica" (Bachelard, 1975: 6768). Es decir, que si se va a plantear el problema del movimiento en la física contemporánea, es necesario, por ejemplo, acceder a un tipo de pensamiento matemático alternativo, esto es: al cálculo de probabilidades, lo cual presupone una ruptura epistemológica con la matemática lineal. De ahí que éste mismo autor afirme que "asimilar esta noción de leyes de azar, enlaces probabilísticos de fenómenos sin enlace real, es la ocupación del pensamiento científico contemporáneo" Bachelard (1985a: 105). Existiría, por lo tanto, una ruptura epistemológica, comprendida como una mutación sicológica, entre el "contexto" epistemológico que dota de sentido a la noción de movimiento en la física clásica y el "contexto" epistemológico que dota de sentido a la noción de movimiento en la física contemporánea. En efecto, para Bachelard es claro que si se quiere plantear el problema del movimiento en su generalidad exacta hay que acceder rápidamente a una especie de instancia de lo posible, ya que la mecánica racional es finalmente la ciencia de los movimientos posibles.

\section{Ideas previas de los estudiantes sobre la noción de movimiento en relación con sus referentes epistemológicos}

Como una primera aproximación a las ideas previas que mantienen los estudiantes de 10 grado al iniciar sus estudios formales de física, presentamos a continuación las gráficas y los análisis correspondientes a la sistematización de los datos obtenidos a partir de la aplicación de la escala Liker a uno de los grupos. Como se había dicho anteriormente, la escala Liker busca identificar el valor de verdad que los estudiantes atribuyen a las diferentes nocio- nes de movimiento que surgen de cuatro paradigmas epistemológicos; a saber: aristotélico, ímpetus, clásico y contemporáneo.

Si bien en las gráficas se mantiene la diferenciación entre los cinco valores que los estudiantes podían atribuir a las proposiciones, para el análisis se han asociado, en cada visión de movimiento, los valores: Completamente de acuerdo (CA) y de Acuerdo $(A)$, en un sólo grupo y lo mismo se ha hecho con los valores: en Desacuerdo (D) y Completamente en desacuerdo (CD). Lo anterior con el fin de mostrar de manera más contundente los hallazgos relacionados con el predominio de las visiones aristotélica y del ímpetus. En efecto, como las valoraciones CA y A presuponen que los estudiantes atribuyen validez a una noción y, por el contrario, las valoraciones $D$ y $C D$ presuponen que los estudiantes no atribuyen validez a esa noción, entonces, las agrupaciones sugeridas muestran porcentualmente con más claridad cual es la posición del grupo de estudiantes.

\subsection{Visión aristotélica (Estudio de caso 1, grupo A.)}

En la gráfica No. 1 se representa la valoración total que los estudiantes de un grupo de 10 grado, de uno de los colegios, atribuye a la noción de movimiento en el paradigma aristotélico. Como se puede notar el grupo de estudiantes atribuye un $29 \%$ de valor de verdad (en el rango de Acuerdo) a las proporciones que constituyen la visión aristotélica sobre el movimiento. Por su parte, el mismo grupo atribuye un $15 \%$ de valor de verdad (en el rango Completamente de acuerdo) a las mismas proposiciones. En oposición a esta valoración el grupo de estudiantes atribuye un $20 \%$ de valor de noverdad (en el rango de Desacuerdo) a las proporciones que constituyen la visión aristotélica sobre el movimiento. Por último, el mismo grupo atribuye un $13 \%$ de valor de no-verdad (en el rango Completamente en desacuerdo) a las proposiciones en 
cuestión. De lo anterior se sigue que el porcentaje de validez que atribuye el grupo a la visión aristotélica del movimiento es del $44 \%$ en contraste con un porcentaje de no-validez del 35\%. Es, pues, mayor el valor de verdad atribuido por el grupo a la visión aristotélica que el valor de no-validez atribuido a la misma. Con lo cual se concluye que es mayor la tendencia a atribuir validez a la visión aristotélica sobre el movimiento en este grupo.

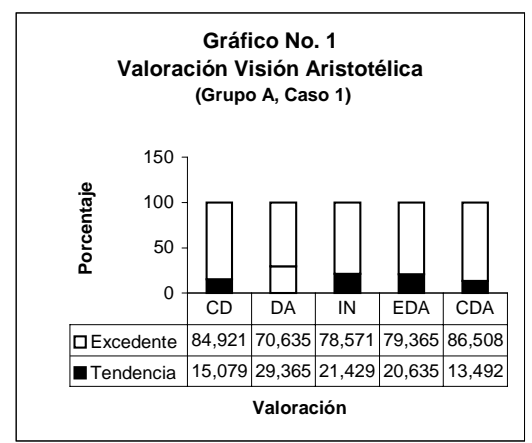

Si tenemos en cuenta las proposiciones de la escala el grupo atribuye, en mayor porcentaje, validez a los siguientes razonamientos sobre el movimiento:

A. Todos los cuerpos permanecen en un lugar que les es natural; por lo tanto, el movimiento es una forma de violencia que aleja al cuerpo de su lugar natural.

B. El movimiento se puede percibir sólo con los sentidos.

C. Todo movimiento tiene un motor externo que lo produce.

\subsection{Visión del impetus (Estudio de caso 1, grupo A)}

En la gráfica No. 2 se representa la valoración total que los estudiantes de un grupo de 10 grado, de uno de los colegios, atribuye a la noción de movimiento en el paradigma del impetus. Como se puede notar el grupo de estudiantes atribuye un $33 \%$ de valor de verdad (en el rango de Acuerdo) a las proporciones que constituyen la visión del Impetus sobre el movimiento. Por su parte, el mismo grupo atribuye un $23 \%$ de valor de verdad (en el rango Completamente de acuerdo) a las mismas proposiciones. En oposición a esta valoración el grupo de estudiantes atribuye un $11 \%$ de valor de no-verdad (en el rango de Desacuerdo) a las proporciones que constituyen la visión del impetus sobre el movimiento. Por último, el mismo grupo atribuye un $3 \%$ de valor de no-verdad (en el rango Completamente en desacuerdo) a las proposiciones en cuestión. De lo anterior se sigue que el porcentaje de validez que atribuye el grupo a la visión del impetus del movimiento es del $56 \%$ en contraste con un porcentaje de no-validez del 14\%. Es pues mayor el valor de verdad atribuido por el grupo a la visión del impetus que el valor de no-validez atribuido a la misma.

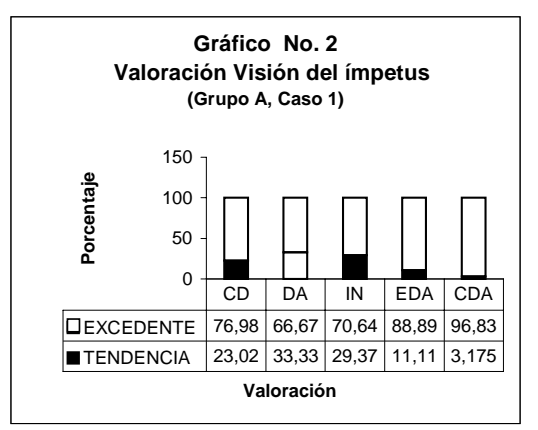

Si tenemos en cuenta las proposiciones de la escala el grupo atribuye, en mayor porcentaje, validez a los siguientes razonamientos sobre el movimiento:

A. Todos los cuerpos tienen en sí mismos una capacidad para moverse.

B. El movimiento es la resultante de por lo menos cuatro variables: peso, forma, posición de la forma y tamaño del móvil.

C. El movimiento es causado por una especie de impulso externo que se impregna en un objeto y le imprime una virtud motriz.

\subsection{Visión clásica (Estudio de caso 1, grupo A)}

En la gráfica No. 3 se representa la valoración total que los estudiantes de un grupo de 10 grado, de uno de los colegios, atribuye a la noción de movimiento en el paradigma de la física clásica. Como se puede notar el grupo de estudiantes atribuye un $16 \%$ de valor de verdad (en el rango de Acuerdo) a las proporciones que constituyen la visión clásica sobre el movimiento. Por su parte, el mismo grupo atribuye un $7 \%$ de valor de verdad (en el rango Completamente de acuerdo) a las mismas proposiciones. En oposición a esta valoración el grupo de estudiantes atribuye un $34 \%$ de valor de noverdad (en el rango en Desacuerdo) a las proporciones que constituyen la visión clásica sobre el movimiento. Por último, el mismo grupo atribuye un $7 \%$ de valor de no-verdad (en el rango Completamente en desacuerdo) a las proposiciones en cuestión. De lo anterior se sigue que el porcentaje de validez que atribuye el grupo a la visión clásica del movimiento es del $23 \%$ en contraste con un porcentaje de no-validez del $43 \%$. Al contrario de las dos visiones anteriores, es pues menor el valor de verdad atribuido por el grupo a la visión clásica que el valor de no-validez atribuido a la misma.

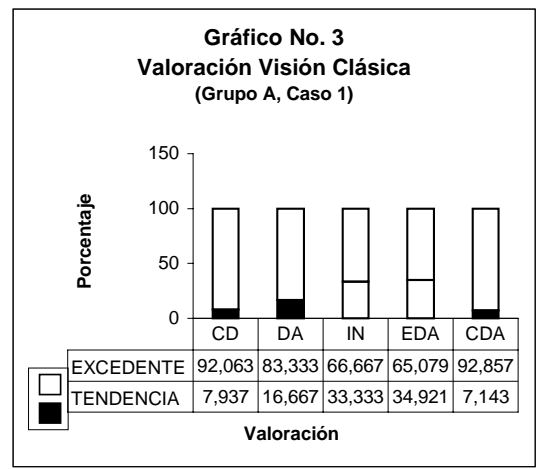

Si tenemos en cuenta las proposiciones de la escala el grupo atribuye, en mayor porcentaje, un valor de no-validez a los siguientes razonamientos sobre el movimiento: 
A. El movimiento se comprende mejor a partir del espacio geométrico que a partir del espacio concreto.

B. El movimiento es un concepto matemático antes que una realidad física.

C. El movimiento es concebido a través del intelecto sin mediación de los sentidos.

\subsection{Visión contemporánea (Estudio de caso 1, grupo A)}

En la gráfica No. 4 se representa la valoración total que los estudiantes de un grupo de 10 grado, de uno de los colegios, atribuye a la noción de movimiento en el paradigma de la física contemporánea. Como se puede notar el grupo de estudiantes atribuye un $30 \%$ de valor de verdad (en el rango de acuerdo) a las proporciones que constituyen la visión contemporánea sobre el movimiento. Por su parte el mismo grupo atribuye un $8 \%$ de valor de verdad (en el rango Completamente de Acuerdo) a las mismas proposiciones. En oposición a esta valoración el grupo de estudiantes atribuye un $18 \%$ de valor de no-verdad (en el rango en desacuerdo) a las proporciones que constituyen la visión contemporánea sobre el movimiento. Por último, el mismo grupo atribuye un $9 \%$ de valor de no-verdad (en el rango Completamente en desacuerdo) a las proposiciones en cuestión. De lo anterior se sigue que el porcentaje de validez que atribuye el grupo a la visión contemporánea del movimiento es del $38 \%$ en contraste con un porcentaje de no-validez del $27 \%$. Al contrario de la visión clásica y manteniendo la misma relación que las dos visiones primeras, es mayor el valor de verdad atribuido por el grupo a la visión contemporánea que el valor de no-validez atribuido a la misma.

Si tenemos en cuenta las proposiciones de la escala el grupo atribuye, en mayor porcentaje, un valor de no-validez a los siguientes razonamientos sobre el movimiento:
A. Cuando decimos: "un rayo de luz", nos referimos a una noción abstracta y no a un dato obtenido por los sentidos.

B. Cuando decimos: "ondas luminosas" nos referimos a una concepción del pensamiento y no a un dato obtenido por los sentidos.

C. Todo fenómeno observable que tiene como origen un "punto luminoso" proviene de un análisis matemático.

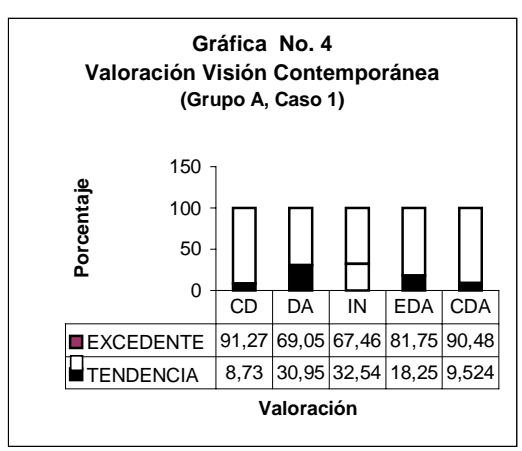

\section{Análisis preliminares de los datos sobre ideas previas de \\ 5. Algunas reflexiones finales acerca de las ideas previas de los estudiantes sobre la noción de movimiento} los estudiantes relacionadas con la noción de movimiento

Si realizamos un análisis comparativo de las cuatro gráficas podemos evidenciar que la noción de movimiento a la que los estudiantes atribuyen en mayor porcentaje de validez es la visión del ímpetus (56\%). En este sentido los estudiantes creen mayoritariamente que el movimiento es una especie de traslación de un objeto de un lugar a otro causado por una fuerza motriz que se encuentra en el mismo objeto, o que en su defecto le es impregnada externamente pero se queda en ella.

Por otra parte, el grupo de estudiantes atribuye en segundo lugar un mayor porcentaje de validez a la noción de movimiento en la física aristotélica (44\%). De esa manera se puede afirmar que los estudiantes creen, en un porcentaje significativo, que el movimiento es una especie de traslación de un objeto de un lugar a otro causado por un motor externo, el cual permanece en contacto con el objeto mientras éste último se mantenga en movimiento. Para el caso de los proyectiles los estudiantes resuelven el problema de la continuidad del contacto entre el móvil y el motor afirmando que el aire continúa trasladando al objeto cuando este pierde contacto con el motor inicial ${ }^{13}$.

Es claro que la noción de movimiento a la que el grupo atribuye menor valor de credibilidad es la correspondiente a la física clásica (23\%). Esto quiere decir que el grupo de estudiantes no cree que la noción de movimiento sea fundamentalmente un concepto matemático cuyo estatuto fundante sea el pensamiento matemático mismo y cuya comprensión dependa del tipo de razonamiento que se ponga en funcionamiento.

Es particularmente llamativo el hecho de que los estudiantes atribuyan un menor grado de valor a los principios epistemológicos relacionados con la noción de movimiento de la visión clásica de la física. Ante todo es importante resaltar que los currículos en educación en ciencias dedican el grado 10 al estudio de la física clásica o mecánica clásica. Lo anterior quiere decir que los profesores de física de 10 grado se enfrentan a un problema pedagógico fundamental, a saber: deben enseñar un concepto como el de movimiento en la mecánica clásica a una población cuyos referentes epistemológicos pertenecen a una visión epistemológicamente "opuesta". En efecto, los estudiantes atribuyen valor de verdad a referentes epistemológicos más cercanos a las formas de razonamiento del sentido común. Cuando

\footnotetext{
${ }^{13}$ Este último dato parece en le análisis del cuestionario y las entrevistas a los grupos de observados.
} 
un estudiante piensa el movimiento, piensa en objetos reales y no necesariamente en objetos matemáticos. Esto introduce un obstáculo epistemológico en el aprendizaje, pues en el lugar de un principio matemático el estudiante coloca una imagen de la vida cotidiana. En lugar de pensar a partir de definiciones, axiomas y teoremas, el estudiante imagina cosas, produce imágenes y sobrepone tales imágenes a los conceptos. De esa manera, el tipo de razonamiento epistemológico que se requiere para comprender un principio matemático y demostrar un teorema es reemplazado por un tipo de razonamiento cuya evidencia primera son las cosas tal y como aparecen en la vida cotidiana.

Pero la mayor dificultad, obviamente no está en que los estudiantes mantengan este tipo de razonamientos epistemológicamente opuestos, sino en el desconocimiento que la enseñanza de las ciencias (particularmente en las aulas) mantiene sobre los mismos. En efecto, por lo general los profesores de física damos por sentado, de manera consciente, que los estudiantes-sujetos ya poseen una suerte de razonamiento que garantiza el aprendizaje de las nociones fundamentales de la mecánica clásica. En ese sentido, el profesor cree que lo que el estudiante-sujeto requiere para aprender es prestar atención y mostrarse interesado en el tema. Los profesores de física no han reflexionado suficientemente sobre el hecho de que en el proceso mismo de la enseña se debe constituir al sujeto del aprendizaje que no es otro que el sujeto epistémico necesario a un nivel de reflexión particular. De no ser así, el profesor sobrepone con su discurso un tipo de razonamiento distinto a las maneras hegemónicas de razonar del estudiante, de tal suerte que el estudiante termina acomodando (por la función de filtro que cumplen las ideas previas) un tipo de razonamiento como el de la mecánica clásica al razonamiento propio de la física aristotélica o al de la física del ímpetus.
Se hace necesario entonces, que los profesores reconozcan cuales son los tipos de razonamiento propios de cada paradigma epistemológico en el que la física ha sido pensada, y con este saber puedan identificar cuales son las formas de razonamiento hegemónicas en sus estudiantes cuando piensan una noción específica. De esa manera los profesores de física podrían favorecer los cambios epistemológicos necesarios para la comprensión de una noción o concepto dentro de una visión claramente particular de la historia de la física.

\section{Estrategias de cambio epistemológico para favorecer la comprensión de la noción de movimiento en la física clásica}

Como ha sido mencionado con anterioridad, en el proyecto se encuentra en desarrollo la puesta en práctica de tres estrategias para favorecer el cambio epistemológico en los estudiantes de física. Estas estrategias son:

A) Un modelo de taller sobre historia y epistemología del concepto de movimiento como apoyo al cambio epistemológico.

B) Un modelo de mini proyectos como apoyo al cambio epistemológico a partir del planteamiento y la resolución de problemas.

C) Un modelo de trabajo con mentefactos.

A continuación presentamos un balance parcial del desarrollo de las dos primeras estrategias, con la seguridad de que cuando se termine el trabajo de campo tendemos nuevos elementos de juicio sobre este proceso.

\subsection{Talleres sobre historia y epistemología del concepto de movimiento como apoyo al cambio epistemológico}

Hasta el momento se han desarrollado 7 talleres. Los 4 primeros ta- lleres se dedicaron a la identificación de formas de razonamiento y conceptos básicos de la física aristotélica. Los tres restantes se han dedicado al análisis crítico del tipo de razonamiento y de los conceptos básicos en la física galileana.

Un criterio fundamental en el desarrollo de los talleres es que los estudiantes se acercan a las formas de razonamiento y a los contenidos específicos de la física a partir del trabajo sobre las fuentes de primera mano.

Un segundo criterio es que los estudiantes identifiquen las dificultades de comprensión que se les presentan cuando leen autores como Aristóteles y Galileo directamente de sus textos.

Un tercer criterio es la flexibilidad en el tiempo y en el abordaje de los temas programados curricularmente.

Un cuarto criterio es la compresión y análisis de los obstáculos y dificultades que aparecen en los estudiantes dadas sus creencias acerca de lo que significa aprender física en la educación media.

\subsubsection{Notas preliminares sobre la aplicación de los talleres}

Posibilitar a los estudiantes el análisis de sus propios preconceptos para que, a través de la confrontación con otros esquemas de pensamiento, puedan renovarse y evolucionar hacia nuevos cambios epistemológicos se puede alcanzar con el estudio de los textos originales. En nuestra experiencia, hemos tomado apartes de la Física de Aristóteles y del libro de Galileo Galilei, Consideraciones y demostraciones matemáticas sobre dos nuevas ciencias. Desde el punto de vista epistemológico, abarcar los textos originales constituye un acierto si se busca que el estudiante por un lado, forme un pensamiento crítico y, por el otro, haga una aproximación vital al pensamiento de quienes formaron las ideas en ciencia. En este marco de 
referencia, "aproximación vital” es abordar tanto las concepciones de los físicos, como los procesos de elaboración del conocimiento que realizaron.

A pesar de que los libros de texto actuales, manuales de enseñanza de la física son una herramienta valiosa a la hora de sintetizar conceptos y mecanizar procesos matemáticos, se enmarcan, en la mayoría de los casos, en una perspectiva histórica y filosófica dogmática y anquilosada que no proporciona al estudiante elementos de juicio para interpretar las rupturas epistemológicas que se han dado en la historia de un mismo concepto. En tales circunstancias, el abordaje de los clásicos se convierte más que en una lectura ilustrativa, en una exigencia si se trata de formar pensamiento crítico.

Al abordar los textos de Aristóteles y Galileo, pudimos evidenciar tres componentes característicos de una formación tradicional en los estudiantes de física:

A. El completo desconocimiento de los textos y sus autores. No es de sorprendernos, generalmente en los primeros años de aproximación al estudio de los fenómenos naturales no hay más referentes que los de la propia experiencia y una que otra lectura esporádica de acontecimientos que hicieron historia. Esto último en el mejor de los casos. Ello nos impele con mayor razón a encontrar en la lectura de los clásicos un campo abierto para el despertar la curiosidad de los estudiantes y la sana confrontación de los maestros con ellos y sus ideas.

B. Una actitud poco receptiva hacia dichas lecturas debido al empuje arrollador de la tradición de la simple transmisión de información. Esto hace que los estudiantes tengan muchos prejuicios frente a la lectura y reflexión y prefieran no involucrarse en estos procesos. Sin embargo, aquí es donde el maestro debe tener la suficiente imaginación como para lograr motivar al estudiantado en el análisis de los textos. Cuando esto se da, sucede que los estudiantes valoran el esfuerzo y pueden lograrse procesos de discusión y análisis muy interesantes.

La posibilidad de confrontar las ideas propias con las de los filósofos y físicos. Este es un aspecto que suele no ser muy tenido en cuenta en los estudios sobre enseñanza-aprendizaje de las ciencias pero que constituye un punto muy importante de nuestra investigación. El propósito es dotar al estudiante de elementos de juicio históricos, filosóficos y científicos para que por su propia experiencia intelectual en la confrontación con los clásicos, pueda diferenciar entre las diversas formas de razonar sobre la naturaleza y, adopte una postura que, desde luego puede ser la de compartir ideas con los filósofos y físicos que se estudian o bien, por el contrario, una postura contraria, pero que le faculte en la difícil tarea de argumentar una posición frente a la forma de ver y aprehender el mundo.

\subsection{Mini proyectos como apoyo al cambio epistemológico}

Hasta el momento se han aplicado 3 miniproyectos cuyo tema integrador es ir "de la noción de movimiento en la física de Aristóteles a la noción de movimiento en la física clásica". Lo anterior se hace a partir del reconocimiento de la importancia que para el cambio de razonamiento tiene el planteamiento y la resolución de problemas.

Las situaciones problemáticas sugeridas a los estudiantes en los tres miniproyectos han sido:
A) Mire, se está moviendo.
B) Oiga, lleva una velocidad.
C) Vea, va a mayor velocidad.

A partir de estas situaciones problemáticas generales los estudiantes inician un proceso de reconocimiento de problemas; especificando qué problemas concretamente les plan- tea a ellos la situación enunciada. Posteriormente, los estudiantes discuten con el profesor los diferentes problemas enunciados por ellos a raíz de la situación general, y formulan un problema de manera adecuada. La definición sobre qué es un problema adecuadamente planteado depende de la concepción de problema que tanto profesor como estudiantes lleguen a concertar. En este caso es importante consultar la bibliografía sobre concepciones de resolución de problemas que circula internacionalmente. Es particularmente importante el momento de los miniproyectos en el cual se producen las interpretaciones del problema construido por los estudiantes. No menos importante son los momentos del miniproyecto en los cuales los estudiantes imaginan, diseñan y socializan las posibles formas de solucionar el problema y llevan a cabo la solución a partir de uno de los procedimientos escogidos.

\subsubsection{Notas preliminares sobre la aplicación de los miniproyectos}

Los estudiantes en principio parecen coincidir con una idea de problemas muy asociada a la que se desarrolla en las clases tradicionales de matemáticas o de física, en donde la interpretación de la situación depende en gran medida de algún tipo de fórmula que se pueda aplicar para resolverlo. Sin embargo, cabe destacar que esta concepción ha venido siendo sometida a la reflexión crítica en la aplicación de los mini proyectos, incluso desde el primer momento en el que cada estudiante debe realizar la sección "reconocimiento del problema" del texto de los mini proyectos, puesto que allí tiene la oportunidad de expresar cuál es el significado de la situación que se está analizando. Así mismo en la sección siguiente: "formulación del problema", se ha podido construir una nueva forma de concebir el problema en física desde una perspectiva que podríamos llamar social y activa ya que desde 
su formulación hasta su procedimiento de solución se ve involucrado directamente el estudiante.

Por lo anterior y hasta el momento del presente escrito la idea de problema como una situación que necesita tener un significado, un sentido, para quien la inventa y la resuelve, subyace en el trabajo de los mini proyectos y ha permitido que los estudiantes se involucren mucho más con la propuesta.

Algunas observaciones del trabajo de los estudiantes en los dos primeros mini proyectos son:

A. Los estudiantes interpretan, en algunas ocasiones, en forma literal las instrucciones a tal punto que en el caso del planteamiento del problema en uno grupo se optó, en primera instancia, por plantear la definición de problema. Aspecto que fue aprovechado para discutir con el profesor sobre el significado que se podía construir sobre la idea de problema. Sin embargo, con la aplicación del segundo mini proyecto se confirman dos tendencias generales de la concepción de problema: por un lado se encuentran los grupos que plantean problemas a la manera usual en el sentido de la enseñanza de las ciencias y más específicamente de las matemáticas. En este tipo de problemas es fundamental la presencia de datos y de un contexto así como de una o varias preguntas.

De otra parte se encuentra la tendencia a concebir el problema como una o varias preguntas. En este caso los estudiantes no especifican demasiado el contexto de la situación y no se anexan datos.

B. En cuanto al ítem de interpretación del problema, se aprecia que éste permite a los estudiantes la reflexión sobre sus propios pensamientos de tal suerte que, en el caso de los estudiantes que plantearon el problema en forma de una o varias preguntas, se constituyó en una oportunidad para que el problema se amplíe, se contextualice mejor y lo que es mejor, comience a tener algún sentido o significado para quienes lo formularon. Por esta razón este aspecto del mini proyecto es de gran importancia.

C. Las explicaciones dadas por los estudiantes acerca de los problemas por ellos formulados han pasado por dos fases generales:

C.1. En primer lugar, los (las) estudiantes argumentan a la fuerza como el "ente" que se requiere para que un cuerpo se mueva. Aunque no se ha profundizado la idea de fuerza para los estudiantes es indistinto hablar de fuerza y de masa, en algunas ocasiones. De igual forma se aduce a la "pérdida" de fuerza como la causante de que un cuerpo se detenga, en algunos casos se hace referencia a una especia de motor interno de los cuerpos que se apaga y este deja de moverse: "al objeto se le acaba la fuerza". En cuanto a las explicaciones sobre la idea de velocidad, ante la pregunta acerca de su significado, los (las) estudiantes de un grupo utilizan la idea de tiempo análoga a la de velocidad; es decir, para explicar cuando un cuerpo lleva una velocidad es porque pasa un determinado tiempo al moverse, pero ello argumentan que la velocidad es lo mismo que el tiempo.

C.2. En segundo lugar, los estudiantes buscan bibliografía y discuten con el profesor acerca de las posibles soluciones a los problemas planteados. En este punto cabe anotar que una vez leen de los textos algunos conceptos de movimiento y de velocidad, los (las) estudiantes centran su atención en la relación distancia tiempo para redefinir su idea de velocidad y así tratar de darle más coherencia a sus explicaciones. Sin embargo, a la hora de estructurar un párrafo explicativo que de solución al problema aún se aprecian recurrencias a términos como aceleración, impulso y energía, que han de ser trabajados en posteriores discusiones.

D. En cuanto a la parte de proceso de solución del problema planteado los recursos utilizados por los (las) estudiantes surgen de sus propias concepciones de movimiento y responden a problemáticas por ellos plateadas. En un grupo, por ejemplo, era importante la forma de los objetos como causa de su mayor o menor posibilidad de moverse, en tal sentido optaron por utilizar objetos esféricos, cubos y largos y redondos como lápices. En otro grupo se utilizaban lápices o esferos y estos se utilizaban como cuerpos que permitían la toma de datos de una mejor forma. En otro grupo se construyeron algunos modelos de aviones para "mirar" cómo volaban y cuánto se mantenían en el aire, así como para dar cuenta por qué se detenían.

\section{Nota final}

Terminado el trabajo de campo (aplicación de mini proyectos y talleres) aplicaremos nuevamente los instrumentos de identificación de ideas previas y pasaremos a analizar si se produjo un cambio epistemológico favorable o no y cuales podrían ser las razones.

\section{Bibliografía}

BachelaRd, G., El nuevo espiritu científico. México: Nueva imagen, 1985a.

La formación el espíritu científico. México: Siglo XXI, 1985b.

La actividad racionalista de la física contemporánea. Buenos Aires: Siglo Veinte, 1975.

- El racionalismo aplicado. Buenos Aires: Piadós, 1978.

Crombie A. C. Historia de la Ciencia de San Agustín a Galileo. Siglos XIII - XVII.

DrIVER, R. Psicología cognoscitiva y esquemas conceptuales de los alumnos. Enseñanza de las Ciencias, 1986, 4, pp. 3-15.

VIENTO, L. "Analising student's reasoning in science: a pragmatic view of theoretical problems". European Journal of Science Education, 1985, 7, pp. 35-46.

Wittrock, C. M. "Procesos de pensamiento de los alumnos". En: La investigación de la enseñanza, III.Barcelona: Piadós, 1990. 